\title{
In situ GaN decomposition analysis by quadrupole mass spectrometry and reflection high-energy electron diffraction
}

\author{
S. Fernández-Garrido, ${ }^{1, a)}$ G. Koblmüller, ${ }^{2}$ E. Calleja, ${ }^{1}$ and J. S. Speck ${ }^{2}$ \\ ${ }^{1}$ ISOM and Dpt. de Ingeniería Electrónica, ETSI Telecomunicación, Universidad Politécnica de Madrid, \\ 28040 Madrid, Spain \\ ${ }^{2}$ Materials Department, University of California, Santa Barbara, California 93106-5050, USA
}

(Received 8 April 2008; accepted 13 June 2008; published online 14 August 2008)

\begin{abstract}
Thermal decomposition of wurtzite (0001)-oriented GaN was analyzed: in vacuum, under active $\mathrm{N}$ exposure, and during growth by rf plasma-assisted molecular beam epitaxy. The GaN decomposition rate was determined by measurements of the Ga desorption using in situ quadrupole mass spectrometry, which showed Arrhenius behavior with an apparent activation energy of $3.1 \mathrm{eV}$. Clear signatures of intensity oscillations during reflection high-energy electron diffraction measurements facilitated complementary evaluation of the decomposition rate and highlighted a layer-by-layer decomposition mode in vacuum. Exposure to active nitrogen, either under vacuum or during growth under N-rich growth conditions, strongly reduced the GaN losses due to GaN decomposition. (C) 2008 American Institute of Physics. [DOI: 10.1063/1.2968442]
\end{abstract}

\section{INTRODUCTION}

III-nitrides have been the subject of intense research since the early 1990s when the first blue light emitting diode ${ }^{1}$ (LED) was developed. Nowadays, devices based on IIInitrides, such as visible and ultraviolet LEDs and laser diodes, or high electron mobility transistors are commercially available. One of the main challenges to achieve these goals was the epitaxial growth of high quality material due to the lack of appropriate substrates. rf plasma-assisted molecular beam epitaxy (PA-MBE) offers several advantages over other epitaxial growth techniques including in situ monitoring of the growth mode and rate by reflection high-energy electron diffraction (RHEED), a low impurity incorporation, and the realization of atomically sharp interfaces.

Though PA-MBE has reached its maturity for the growth of III-nitrides, a more complete analysis of the growth dynamics and thermal effects has yet to be accomplished. The growth of GaN by PA-MBE is a metastable process because $\mathrm{GaN}$ is thermodynamically unstable at pressures typical in the molecular beam regime $\left(<10^{-4}\right.$ Torr $){ }^{2,3}$ To grow GaN, the forward reaction rate $\left(v_{f}\right)$

$$
\mathrm{Ga} \text { (liquid or vapor) }+1 / x \mathrm{~N}_{x}^{*}(\text { vapor }) \rightarrow \mathrm{GaN}(\text { solid }),
$$

where $\mathrm{N}_{x}^{*}$ represents any of the possible reactive nitrogen species, must be higher than the $\mathrm{GaN}$ decomposition rate or reverse reaction $\left(v_{r}\right)$

$$
\mathrm{GaN}(\text { solid }) \rightarrow \mathrm{Ga} \text { (liquid or vapor) }+1 / 2 \mathrm{~N}_{2} \text { (vapor) } \text {. }
$$

The forward reaction rate is determined by the fluxes of the reactive species whose values are limited by the molecular beam regime, while the reverse reaction rate depends on the substrate temperature through the kinetic barrier for $\mathrm{GaN}$ decomposition. ${ }^{2,3}$ Thus, for a given $\mathrm{Ga} / \mathrm{N}$ flux ratio, the maximum growth temperature is given by $v_{f}-v_{r}$.

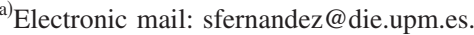

Therefore, one of the key issues toward the development of a high temperature growth diagram and toward improvements in the quality of the GaN grown by PA-MBE is to understand the mechanism behind $\mathrm{GaN}$ decomposition and its possible dependence on the growth parameters.

This work reports on the temperature dependence of wurtzite (0001)-oriented $\mathrm{GaN}$ decomposition (in the range from 720 to $805^{\circ} \mathrm{C}$ ) under different conditions: (i) in vacuum (at $\mathrm{N}_{2}$ partial pressure of $1.5 \times 10^{-5}$ Torr), (ii) under active $\mathrm{N}$ exposure, and (iii) during standard $\mathrm{GaN}$ growth conditions ${ }^{4}$ (Ga-rich and N-rich conditions). Two complementary techniques were employed to determine the GaN decomposition rate: first, line-of-sight quadrupole mass spectrometry (QMS) to measure the desorbed $\mathrm{Ga} \mathrm{flux}^{5,6}$ at elevated temperatures, and second RHEED intensity measurements to gain additional knowledge about the decomposition mode.

\section{EXPERIMENT}

The studies were carried out in a Varian Gen-II MBE system equipped with a Vecco Unibulb rf-plasma $\mathrm{N}$ source and two Ga Knudsen cells. During all experiments the $\mathrm{N}_{2}$ partial pressure in the MBE system was $1.5 \times 10^{-5}$ Torr. As substrates we used 2 in. (0001) GaN templates $(3.6 \mu \mathrm{m}$ thick) grown by metal-organic chemical vapor deposition on $c$-plane sapphire (Lumilog), which were outgassed for $1 \mathrm{~h}$ at $400{ }^{\circ} \mathrm{C}$ prior to the experiments. The substrate temperature was measured using an optical pyrometer calibrated to the melting point of $\mathrm{Al}\left(660^{\circ} \mathrm{C}\right)$. Measurements of the dependence of the desorbing Ga flux on substrate temperature during GaN decomposition in vacuum, under active $\mathrm{N}$ exposure, and during PA-MBE growth were performed using the QMS technique, which is described in detail elsewhere. ${ }^{5,6}$ Both the QMS response flux and all other impinging fluxes are given in (0001) GaN growth units in $\mathrm{nm} / \mathrm{min}^{4,6}$ We note that 1 $\mathrm{nm} / \mathrm{min}$ is equivalent to $0.064 \mathrm{ML} / \mathrm{s}$ (ML denotes monolayer), where one $\mathrm{ML}$ of $\mathrm{GaN}$ corresponds to $c / 2$ 

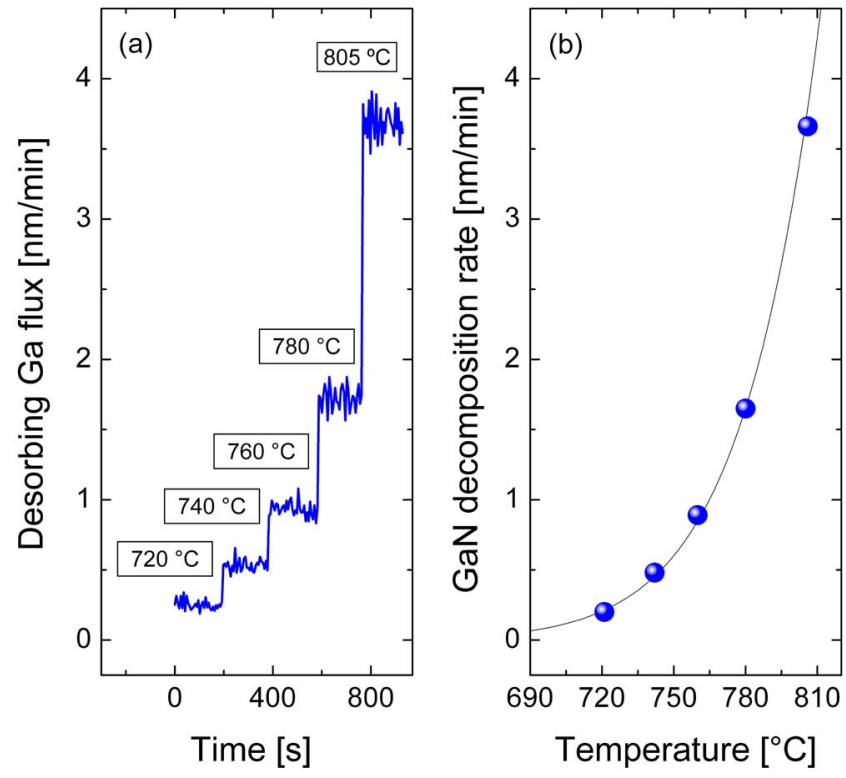

FIG. 1. (Color online) (a) Desorbing Ga flux assessed by QMS, $\Phi_{\mathrm{QMS}}$, during $\mathrm{GaN}$ decomposition in vacuum at different substrate temperatures. (b) Dependence of the GaN decomposition rate on substrate temperature in vacuum as derived from $\Phi_{\mathrm{QMS}}$. The solid line corresponds to the fit to $\mathrm{Eq}$. (3).

$=0.259 \mathrm{~nm}$ or $1.14 \times 10^{15} \mathrm{GaN} / \mathrm{cm}^{2}$ areal density along the (0001) direction. The RHEED pattern and the intensity profile were monitored using a charge-coupled device detection system (k-Space Associates). To ensure reproducible data, recovery of the GaN surface was achieved by growing a few MLs of GaN under Ga-rich conditions slightly below the limit of $\mathrm{Ga}$ droplet formation ${ }^{4}$ prior to the initiation of each experiment.

\section{RESULTS}

\section{A. GaN decomposition in vacuum}

Since GaN decomposes congruently in vacuum, ${ }^{7}$ the desorbing Ga flux measured by QMS ( $\left.\Phi_{\mathrm{QMS}}\right)$ provided a direct measurement of the GaN decomposition rate. Figure 1(a) depicts the steady-state desorbing Ga flux recorded at substrate temperature intervals of $\sim 20^{\circ} \mathrm{C}$ between 720 and $805^{\circ} \mathrm{C}$. As shown in Fig. 1(b), the GaN decomposition rate derived from the desorbing Ga flux increased exponentially with temperature from nearly zero at $720^{\circ} \mathrm{C}$ to above $\sim 3.5 \mathrm{~nm} / \mathrm{min}$ (i.e., $0.2 \mu \mathrm{m} / \mathrm{h}$ ) at $805^{\circ} \mathrm{C}$. The onset temperature and the rates for thermal decomposition compare favorably with previous results from laser reflectivity. ${ }^{8}$

During GaN decomposition a streaky RHEED pattern was observed with clear intensity oscillations along the [11̄̄0] azimuth for temperatures above $740{ }^{\circ} \mathrm{C}$ [Fig. 2(a)]. With increasing temperature the period of oscillations $\tau_{\mathrm{osc}}$ became gradually shorter. Since each oscillation period corresponds to the decomposition of one ML of $\mathrm{GaN}$, we were able to determine the decomposition rate for each temperature from the analysis of the RHEED intensity evolution. The decomposition rate given by $1 / \tau_{\text {osc }}$ was nearly zero below $740{ }^{\circ} \mathrm{C}$ and increased exponentially with temperature exceeding a rate of $\sim 3.5 \mathrm{~nm} / \mathrm{min}$ at $805{ }^{\circ} \mathrm{C}$ [Fig. 2(b)], in
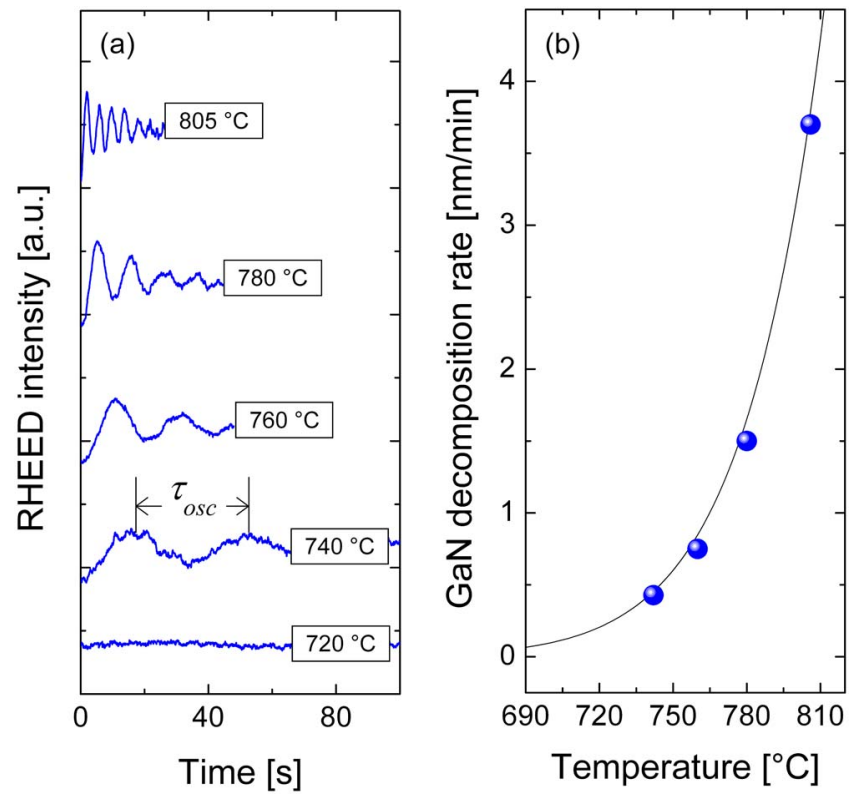

FIG. 2. (Color online) (a) RHEED intensity oscillations during GaN decomposition in vacuum at different temperatures. (b) Dependence of the GaN decomposition rate on substrate temperature in vacuum as derived from the RHEED intensity oscillation periods, $\tau_{\text {osc }}$. The solid line corresponds to the fit to Eq. (3).

close agreement with the values determined by QMS. The observation of intensity oscillations can be further attributed to a two-dimensional (2D) layer-by-layer decomposition mode, similar to the typical growth rate oscillations during GaN growth. ${ }^{9}$ In contrast, Grandjean et al. ${ }^{8}$ also reported a streaky RHEED pattern during GaN decomposition but observed no intensity oscillations, suggesting rather a step-flow decomposition mode. We further note that the streaky RHEED pattern persisted well beyond the damping of the intensity oscillations (at least for others $60 \mathrm{~s}$ until the next surface recovery cycle), indicating the continuity of the 2D decomposition mode with no roughening of the GaN surface.

The Arrhenius plot of data points for the GaN decomposition rate (as evaluated by both QMS and RHEED) (Fig. 3) revealed a temperature dependence for the $\mathrm{GaN}$ decomposition rate, $\Phi_{D}$, defined as

$$
\Phi_{D}=A \exp \left(-E_{D} / k_{B} T\right),
$$

where the exponential prefactor, $A$, was fitted to $(1.6 \pm 0.1)$ $\times 10^{15} \mathrm{~nm} / \mathrm{min}$ and the apparent activation energy for GaN thermal decomposition was best fitted to $E_{D}=3.1 \pm 0.1 \mathrm{eV}$. This is in good agreement with the values reported in literature, ranging from 3.1 to $3.6 \mathrm{eV}^{7,8,10,11}$

\section{B. GaN losses during PA-MBE growth and in vacuum under active $\mathrm{N}$ exposure}

The GaN losses as a result of $\mathrm{GaN}$ decomposition under standard GaN growth conditions, as defined by the different growth conditions within the previously developed $\mathrm{GaN}$ growth diagram, ${ }^{4}$ and in vacuum under active $\mathrm{N}$ exposure were also studied as a function of the substrate temperature. In particular, we investigated (i) intermediate Ga-rich conditions, where the GaN surface is terminated by a $\mathrm{Ga}$ 


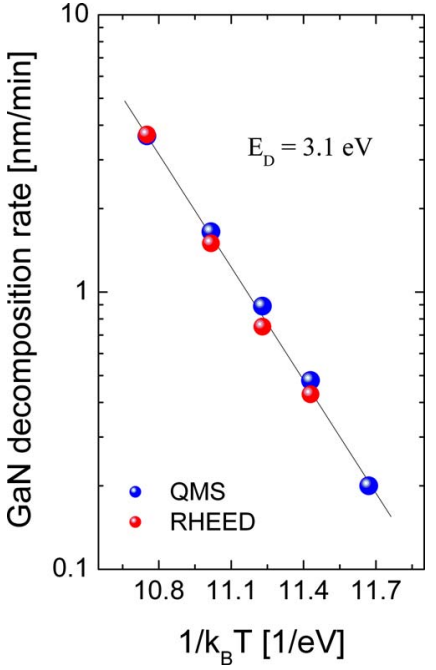

FIG. 3. (Color online) GaN decomposition rate in vacuum (as derived from both the desorbing Ga flux assessed by QMS and the RHEED intensity oscillation periods) as a function of $1 / k_{B} T$. The activation energy for GaN decomposition is $E_{D}=3.1 \pm 0.1 \mathrm{eV}$.

adlayer $^{4-6} \quad\left(\Phi_{\mathrm{Ga}}=7.0, \quad 5.6\right.$, and $5.0 \mathrm{~nm} / \mathrm{min}$ for $\Phi_{\mathrm{N}}$ $=5.0 \mathrm{~nm} / \mathrm{min}$ and $\Phi_{\mathrm{Ga}}=10$ and $8.0 \mathrm{~nm} / \mathrm{min}$ for $\Phi_{\mathrm{N}}$ $=7.0 \mathrm{~nm} / \mathrm{min})$, (ii) N-rich conditions $\left(\Phi_{\mathrm{Ga}}=4.0,3.0\right.$, and $1.0 \mathrm{~nm} / \mathrm{min}$ for $\Phi_{\mathrm{N}}=5.0 \mathrm{~nm} / \mathrm{min}$ and $\Phi_{\mathrm{Ga}}=6.0,5.0$, and $4.0 \mathrm{~nm} / \mathrm{min}$ for $\Phi_{\mathrm{N}}=7.0 \mathrm{~nm} / \mathrm{min}$ ), and (iii) exposure to active $\mathrm{N}$ without impinging $\mathrm{Ga}\left(\Phi_{\mathrm{Ga}}=0, \Phi_{\mathrm{N}}=5.0\right.$ and $7 \mathrm{~nm} / \mathrm{min}$ ). In the following, to describe the actual GaN losses under these dynamic conditions, we introduce the term effective GaN decomposition rate defined as the flux of $\mathrm{Ga}$ or $\mathrm{N}$ atoms actually desorbed after $\mathrm{GaN}$ decomposition.

Under intermediate Ga-rich growth conditions a streaky RHEED pattern with intensity oscillations indicative of a 2D growth mode was observed for all temperatures, while under N-rich conditions we found a spotty-to-streaky transition with increasing growth temperature. This corresponds to a transition in growth mode from three-dimensional to $2 \mathrm{D}$ layer-by-layer growth as recently highlighted by the sustainable 2D surface morphologies of layers grown under N-rich conditions in the GaN thermal decomposition regime. ${ }^{12,13}$ During GaN decomposition under active $\mathrm{N}$ exposure, the RHEED pattern became gradually spotty indicating progressive roughening of the GaN surface. This inhibited the assessment of the effective $\mathrm{GaN}$ decomposition rate under active $\mathrm{N}$ exposure using RHEED intensity analysis, in contrast to the layer-by-layer-like decomposition observed in vacuum under static conditions (i.e., no impinging fluxes).

For those growth conditions where clear RHEED intensity oscillations were observed, we were able to investigate the effect of the $\mathrm{Ga} / \mathrm{N}$ flux ratio on the effective $\mathrm{GaN}$ decomposition rate. Figure 4(a) shows the actual growth rate given by $1 / \tau_{\text {osc }}$ (where $\tau_{\text {osc }}$ is the RHEED intensity oscillation period) at $770{ }^{\circ} \mathrm{C}$ as a function of the impinging $\mathrm{Ga}$ flux for two different active $\mathrm{N}$ fluxes $\left(\Phi_{\mathrm{N}}=5.0\right.$ and $\left.7.0 \mathrm{~nm} / \mathrm{min}\right)$. In both cases, the growth rate steadily increased until flux stoichiometry was achieved $\left(\Phi_{\mathrm{Ga}}=\Phi_{\mathrm{N}}\right)$. An estimation of the effective GaN decomposition rate was made assuming a unity sticking coefficient for the impinging $\mathrm{Ga}$ and $\mathrm{N}$ atoms for N-rich and Ga-rich growth conditions, respectively [Fig.

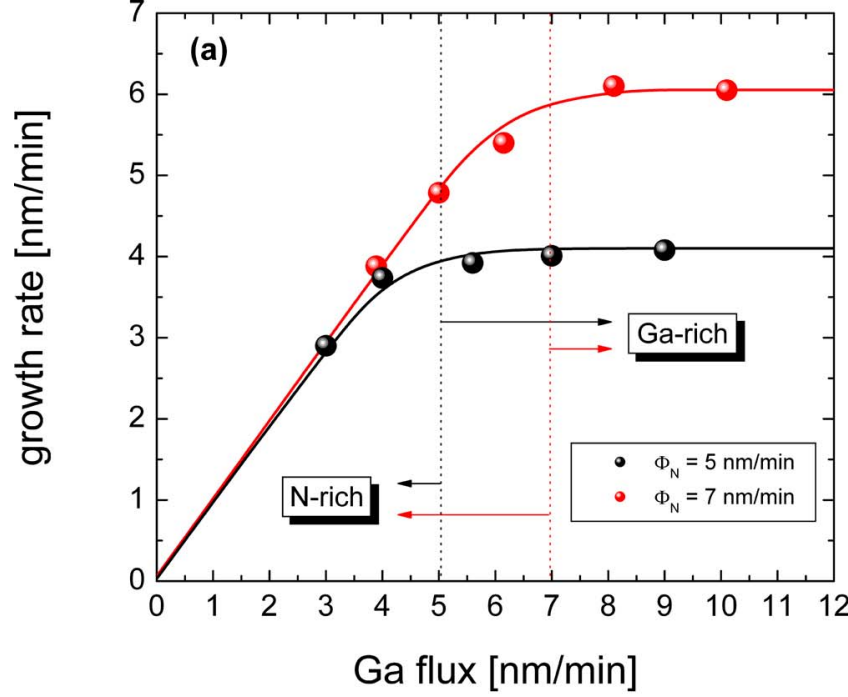

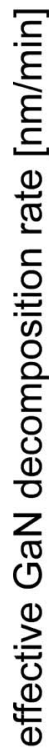

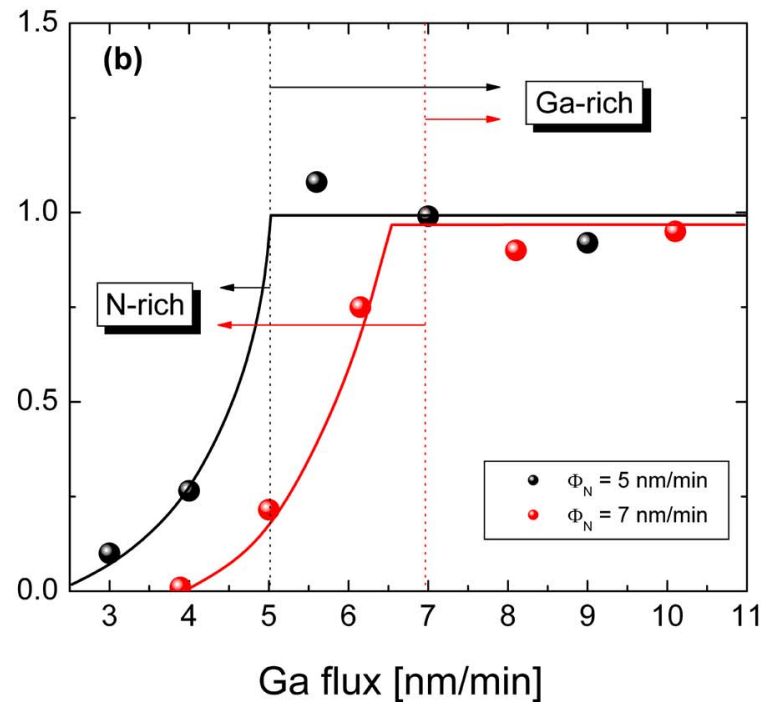

FIG. 4. (Color online) GaN growth (a) and effective decomposition (b) rates at $770{ }^{\circ} \mathrm{C}$ as a function of the impinging Ga flux for two different active $\mathrm{N}$ fluxes, 5.0 and $7.0 \mathrm{~nm} / \mathrm{min}$. The growth rate was assessed by RHEED intensity oscillations and the effective $\mathrm{GaN}$ decomposition rate was derived from the reduction of the nominal growth rate given by $\Phi_{\mathrm{N}}$ or $\Phi_{\mathrm{Ga}}$ for Ga-rich or N-rich growth conditions, respectively.

4(b)]. Thus, the effective $\mathrm{GaN}$ decomposition rate was evaluated as the nominal growth rate (given by the fixed impinging fluxes $\Phi_{\mathrm{N}}$ or $\Phi_{\mathrm{Ga}}$ for Ga-rich or N-rich growth conditions, respectively) minus the actual growth rate derived from the RHEED intensity oscillation periods [Fig. 4(a)]. For a given active $\mathrm{N}$ flux, the effective $\mathrm{GaN}$ decomposition rate steadily increased with the impinging Ga flux under N-rich conditions and saturated near $1 \mathrm{~nm} / \mathrm{min}$ once flux stoichiometry was achieved. For a given Ga flux within the N-rich regime, the effective decomposition rate decreased with the active $\mathrm{N}$ flux. These results suggest a strong reduction of the effective GaN decomposition rate under N-rich conditions, toward higher $\mathrm{N}$ excess. Also, it is obvious that for slightly Ga-rich conditions the effective decomposition rate is independent of the $\mathrm{Ga} / \mathrm{N}$ flux ratio (both by excess $\mathrm{Ga}$ and active $\mathrm{N})$ and matches the GaN decomposition rate determined un- 


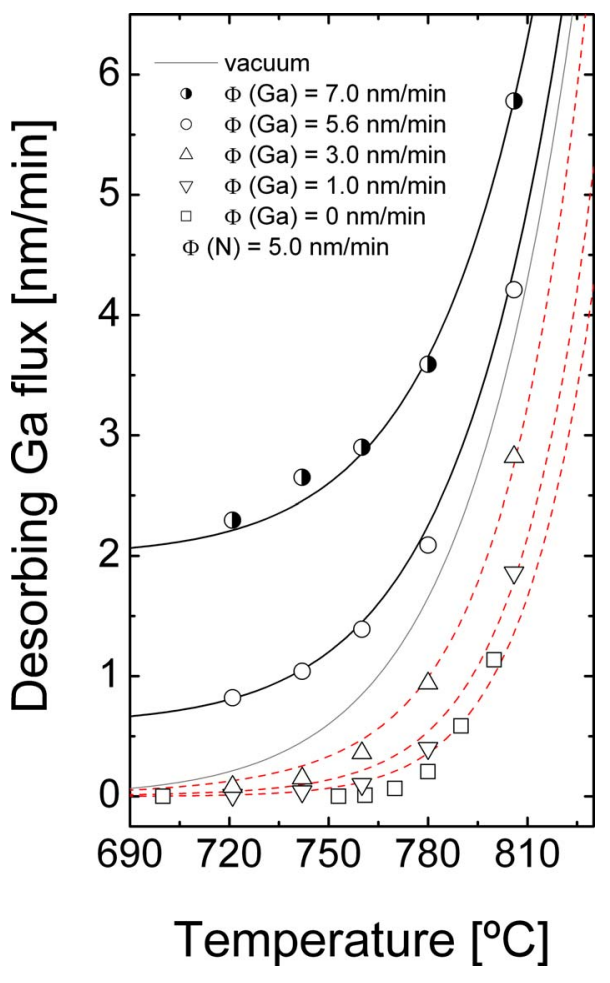

FIG. 5. (Color online) Dependence of the desorbing Ga flux $\left(\Phi_{\mathrm{QMS}}\right)$ on substrate temperature under slightly Ga-rich growth conditions $\left(\Phi_{\mathrm{Ga}}=7.0\right.$ and $5.6 \mathrm{~nm} / \mathrm{min}, \Phi_{\mathrm{N}}=5.0 \mathrm{~nm} / \mathrm{min}$ ) and under excess active $\mathrm{N}$ conditions $\left(\Phi_{\mathrm{Ga}}=3.0,1.0\right.$, and $\left.0 \mathrm{~nm} / \mathrm{min} ; \Phi_{\mathrm{N}}=5.0 \mathrm{~nm} / \mathrm{min}\right)$. The black solid lines correspond to the plot of Eq. (4) for slightly Ga-rich conditions while the dashed lines are guides to the eyes. The desorbing Ga flux during $\mathrm{GaN}$ decomposition in vacuum was also included for comparison (gray solid line).

der static conditions (in vacuum, no growth). Nevertheless, we note that slight deviations between the actual effective GaN decomposition rates and those values shown in Fig. 4(b) are expected since the Ga sticking coefficient could be lower than unity for the studied range of temperatures and could depend on the specific active $\mathrm{N}$ excess as further discussed in Sec. IV.

To corroborate RHEED results, the effective $\mathrm{GaN}$ decomposition rate during PA-MBE growth and in vacuum under active $\mathrm{N}$ exposure was also investigated by QMS. Figure 5 shows the desorbed Ga flux, $\Phi_{\mathrm{QMS}}$, under these dynamic conditions. As expected, in all cases $\Phi_{\mathrm{QMS}}$ was found to increase exponentially with substrate temperature. A fitted curve for $\Phi_{\mathrm{QMS}}$ during $\mathrm{GaN}$ decomposition in vacuum (i.e., $\Phi_{D}$, no impinging fluxes) as shown in Figs. 1(b) and 2(b). is also included for reference. Under slightly Ga-rich growth conditions, $\Phi_{\mathrm{OMS}}$ was larger than $\Phi_{D}$, due to the typical excess $\mathrm{Ga}$ desorbing from the surface, ${ }^{4}$ as further discussed below. During $\mathrm{N}$-rich growth and under active $\mathrm{N}$ exposure $\Phi_{\mathrm{QMS}}$ was well below $\Phi_{D}$ and decreased with lower $\mathrm{Ga} / \mathrm{N}$ flux ratios. These results point thus to a significant reduction of the effective $\mathrm{GaN}$ decomposition rate under active $\mathrm{N}$ excess (i.e., during $\mathrm{N}$-rich growth or under active $\mathrm{N}$ exposure) in good agreement with the results derived from the analysis of the RHEED intensity evolution.

\section{DISCUSSION}

To explain these deviations from the static conditions, we stress that under the dynamic conditions the desorbed Ga flux may not only arise from the Ga adatoms desorbing during GaN decomposition but also from partial desorption of the impinging Ga flux during growth. The latter holds especially true not only for the excess Ga under Ga-rich conditions but also for excess active $\mathrm{N}$ conditions, where the reduced Ga surface residence lifetime at elevated temperatures may limit the incorporation probability. For the Ga adatoms segregated to the surface under decomposition they can be either desorbed (as in the Ga-rich case) or reincorporated into the layer (as in the $\mathrm{N}$-rich case) via reaction with active $\mathrm{N}$ thus reducing the effective GaN decomposition rate.

Under slightly Ga-rich growth conditions the active $\mathrm{N}$ is assumed to be entirely consumed by the impinging Ga flux. ${ }^{4}$ Thus, under steady-state conditions, if there is no metal accumulation on the surface (as in form of Ga droplets) and the effective $\mathrm{GaN}$ decomposition rate is unaffected by the impinging fluxes, the desorbing Ga flux should be given by

$$
\Phi_{\mathrm{QMS}}=\Phi_{\mathrm{Ga}}-\Phi_{\mathrm{N}}+\Phi_{D},
$$

meaning that $\Phi_{\mathrm{QMS}}$ is given by the excess of the impinging $\mathrm{Ga}$ flux plus the $\mathrm{GaN}$ decomposition rate measured in vacuum. As evident in Fig. 5, this properly reproduces the experimental data without any fitting. Thus, we conclude that the effective GaN decomposition rate under slightly Ga-rich conditions is identical to the $\mathrm{GaN}$ decomposition rate found in vacuum (i.e., $\Phi_{D}$ ). Slightly Ga-rich conditions therefore result in unmodified $\mathrm{GaN}$ losses, as long as the $\mathrm{GaN}$ surface is not saturated with liquid Ga. The Ga coverage under these investigated temperatures and Ga fluxes was measured to be below $1 \mathrm{ML},{ }^{12}$ far away from the formation boundary of liquid Ga droplets. Liquid Ga droplets were considered to act as a catalyst for GaN decomposition ${ }^{14}$ and were found to be correlated with the GaN decomposition enhancement observed in $\mathrm{H}_{2}$ near atmospheric pressure. ${ }^{15}$ Furthermore, given that the absolute value of the $\mathrm{GaN}$ decomposition rate approaches the N-limited $\mathrm{GaN}$ growth rate (i.e., $5.0 \mathrm{~nm} / \mathrm{min}$ ) at $810^{\circ} \mathrm{C}$, this temperature marks an upper limit for the successful growth of $\mathrm{GaN}$ for this fixed nitrogen flux and if exceeded results in thermal etching. It is therefore expected that higher active nitrogen fluxes will increase the Ga incorporation rate and thus enable growth at even higher temperatures.

In contrast, the results derived from both RHEED and QMS demonstrate that the effective GaN decomposition rate is strongly reduced under active $\mathrm{N}$ excess. From a thermodynamic point of view, the reduction of the effective GaN decomposition rate under such conditions can be explained considering Le Chatelier's principle. For a given temperature and impinging $\mathrm{Ga}$ flux, an increase in the active $\mathrm{N}$ flux means a higher concentration of the reactives in Eq. (1). This yields a shift of the reaction in the opposite direction to counteract the imposed change as long as there is Ga available. Thus, the ratio between the $\mathrm{Ga}$ adatoms that are reincorporated into the layer (after GaN decomposition) and those that are desorbed from the surface is increased for 


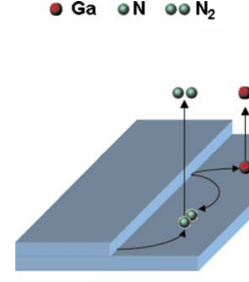

(a) (b)

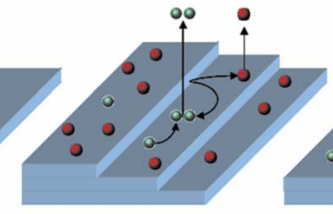

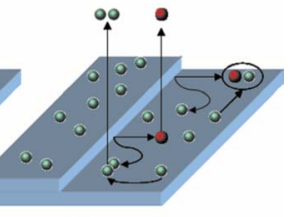

(c)
FIG. 6. (Color online) Schematic of the GaN surface during GaN decomposition in vacuum (a), under slightly Ga-rich growth conditions (b), and under active $\mathrm{N}$ excess conditions (i.e., during $\mathrm{N}$-rich growth or in vacuum under active $\mathrm{N}$ exposure) (c). The mechanistic desorption and reincorporation (for active $\mathrm{N}$ excess conditions) pathways for the decomposed species are shown in each case. The impinging fluxes in (b) and (c) are not depicted for clarity purposes.

higher active $\mathrm{N}$ fluxes resulting in a reduction of the effective GaN decomposition rate. These results highlight the possibility to grow $\mathrm{GaN}$ under N-rich conditions at higher temperatures than those expected from the $\mathrm{GaN}$ decomposition rate measured in vacuum, paving the way to an alternative approach to grow high quality material by PA-MBE. ${ }^{13}$

From a surface kinetic point of view, the differences between $\mathrm{GaN}$ losses in vacuum, Ga-rich, and N-rich conditions can be understood by the capture probability and surface diffusion of the decomposed species with respect to the excess versus deficiency of $\mathrm{N}$ atoms (see Fig. 6). This is primarily based on the assumption that the decomposed species (i.e., those $\mathrm{Ga}$ and $\mathrm{N}$ atoms resulting from $\mathrm{GaN}$ decomposition) follow the mechanistic desorption pathway from solid to vapor via an adsorbed surface state

$$
\begin{aligned}
& \mathrm{GaN}(\text { solid }) \rightarrow \mathrm{Ga} \text { (adsorbed })+\mathrm{N}(\text { adsorbed }), \\
& \mathrm{Ga}(\text { adsorbed }) \rightarrow \mathrm{Ga}(\text { vapor }), \\
& \left.\mathrm{N}(\text { adsorbed })+\mathrm{N} \text { (adsorbed }) \rightarrow \mathrm{N}_{2} \text { (vapor }\right) .
\end{aligned}
$$

The proof for these intermediate adsorbed surface states of segregated $\mathrm{Ga}$ and $\mathrm{N}$ adatoms is directly provided by the reduction in the effective $\mathrm{GaN}$ decomposition rate due to their reincorporation under excess $\mathrm{N}$ conditions. If $\mathrm{GaN}$ decomposes directly from the solid to the vapor phase, the effective decomposition rate would not have been influenced at all by active nitrogen, yielding constant rates throughout all possible growth conditions. It is important to note that the segregated $\mathrm{Ga}$ atoms from $\mathrm{GaN}$ decomposition cannot accumulate as liquid $\mathrm{Ga}$ (in the form of droplets), since the rate for thermal $\mathrm{GaN}$ decomposition is far below the desorption rate from a liquid $\mathrm{Ga}$ reservoir. ${ }^{4,9}$

In the simple case of decomposition into vacuum or under stoichiometric flux conditions, the rate of thermal GaN losses is therefore determined by the immediate evaporation of the segregated $\mathrm{Ga}$ and $\mathrm{N}$ adatoms due to the lack of excess $\mathrm{Ga}$ or $\mathrm{N}$ atoms provided to the surface.

However, under N-rich conditions the capture and reincorporation probabilities between segregated $\mathrm{Ga}$ and $\mathrm{N}$ adatoms are increased, partly due to the higher density of excess $\mathrm{N}$ and the modified surface diffusion behavior under these conditions. The latter can be understood by the fact that $\mathrm{N}$ adatoms are expected to be kinetically stabilized on the sur- face, although they are thermodynamically highly unstable against desorption as $\mathrm{N}_{2}$ molecules. According to Zywietz et al.,${ }^{16}$ the migration of $\mathrm{N}$ adatoms on a surface saturated with excess $\mathrm{N}$ is a highly activated process (i.e., with a calculated diffusion barrier of $1.4 \mathrm{eV}),{ }^{16}$ such that the subsequent formation and evaporation rate of $\mathrm{N}_{2}$ may become smaller than the capture rate by faster moving Ga adatoms. Consequently, the mean diffusion length of the Ga adatoms is limited by the capture with $\mathrm{N}$ adatoms, explaining the observed reduction in desorption of $\mathrm{Ga}$ adatoms.

Under $\mathrm{N}$ deficient (i.e., Ga-rich) conditions, the surface kinetics behaves in the opposite way. First, no excess active $\mathrm{N}$ is available to be captured by the segregated $\mathrm{Ga}$ adatoms since all impinging $\mathrm{N}$ atoms are entirely consumed by the supplied Ga flux. ${ }^{4}$ Also, on a Ga-rich GaN surface the diffusion barrier of $\mathrm{N}$ adatoms is reduced (i.e., $0.2 \mathrm{eV}$ as in the extreme case of a liquid $\mathrm{Ga}$ bilayer terminating the surface under heavy Ga-rich conditions). ${ }^{17}$ This results in higher migration of $\mathrm{N}$ adatoms and formation rates of volatile $\mathrm{N}_{2}$, which in turn means much lower capture probability with $\mathrm{Ga}$ adatoms and enhanced effective GaN decomposition rates. This may also explain the recent findings that liquid Ga (either in the form of a bilayer or droplets) can even catalyze GaN decomposition. ${ }^{14}$

Considering the similarity between the decomposition rates into vacuum (i.e., no growth) and the effective decomposition rates observed under slightly Ga-rich conditions, we suggest that in the present study surface diffusion plays an inferior role due to the low amounts of excess Ga. Recent studies showed that the Ga adlayer coverage involved under these slightly Ga-rich conditions at high temperature is much less than $1 \mathrm{ML},{ }^{12}$ which is expected to have not too significant effects on the $\mathrm{N}$ adatom diffusion. ${ }^{18}$ Increases in Ga flux and larger adlayer coverages $(>2 \mathrm{ML})$ would be required to obtain more substantial deviations between the decomposition rate into vacuum and the effective decomposition rate during Ga-rich growth.

\section{CONCLUSIONS}

GaN decomposition was studied in situ by RHEED and QMS in vacuum with and without active $\mathrm{N}$ exposure, and during GaN PA-MBE growth (under slightly Ga-rich and $\mathrm{N}$-rich growth conditions). RHEED intensity oscillations during GaN decomposition in vacuum revealed that $\mathrm{GaN}$ decomposes by a layer-by-layer mode. From both the period of RHEED intensity oscillations and the desorbing Ga flux assessed by QMS, the temperature dependent rates for $\mathrm{GaN}$ decomposition were determined and an apparent activation energy of $3.1 \mathrm{eV}$ was deduced. Growth under slightly Garich conditions yielded an effective $\mathrm{GaN}$ decomposition rate identical to the GaN decomposition rate measured in vacuum. In contrast, under $\mathrm{N}$-rich growth conditions or exposing the GaN surface to active $\mathrm{N}$, the effective $\mathrm{GaN}$ decomposition rate was substantially suppressed. This effect was found to be enhanced for higher active $\mathrm{N}$ excess due to the increase in $\mathrm{Ga}$ capture probability by $\mathrm{N}$ adatoms. 


\section{ACKNOWLEDGMENTS}

Thanks are due to A. Hirai, C. Gallinat, A. Corrion, and C. Poblenz for fruitful discussions (all UCSB). The authors gratefully acknowledge support from AFOSR (Donald Silversmith, Program Manager). This work made use of the MRL Central Facilities and was also partially supported by the Spanish Ministry of Education (MAT2004-2875, NAN04/09109/C04/2, Consolider-CSD 2006-19, and FPU program) and the Community of Madrid (GR/MAT/0042/ 2004 and S-0505/ESP-0200).

${ }^{1}$ S. Nakamura and G. Fasol, The Blue Laser Diode-GaN Light Emitters and Lasers (Springer, Berlin, 1997).

${ }^{2}$ N. Newman, J. Ross, and M. Rubin, Appl. Phys. Lett. 62, 1242 (1993).

${ }^{3}$ N. Newman, J. Cryst. Growth 178, 102 (1997).

${ }^{4}$ B. Heying, R. Averbeck, L. F. Chen, E. Haus, H. Riechert, and J. S. Speck, J. Appl. Phys. 88, 1855 (2000).

${ }^{5}$ G. Koblmüller, R. Averbeck, H. Riechert, and P. Pongratz, Phys. Rev. B 69, 035325 (2004).

${ }^{6}$ J. S. Brown, G. Koblmüller, F. Wu, R. Averbeck, H. Riechert, and J. S
Speck, Appl. Phys. Lett. 99, 074902 (2006).

${ }^{7}$ Z. A. Munir and A. W. Searcy, J. Chem. Phys. 42, 4223 (1965).

${ }^{8}$ N. Grandjean, J. Massies, F. Semond, S. Yu. Karpov, and R. A. Talalaev, Appl. Phys. Lett. 74, 1854 (1999).

${ }^{9}$ C. Adelmann, J. Brault, G. Mula, B. Daudin, L. Lymperakis, and J. Neugebauer, Phys. Rev. B 67, 165419 (2003).

${ }^{10}$ R. Groh, G. Gerey, L. Bartha, and J. I. Pankove, Phys. Status Solidi 26, 353 (1974).

${ }^{11}$ R. Held, D. E. Crawford, A. M. Johnston, A. M. Dabiran, and P. I. Cohen, Surf. Rev. Lett. 5, 913 (1998).

${ }^{12}$ G. Koblmüller, S. Fernández-Garrido, E. Calleja, and J. S. Speck, Appl. Phys. Lett. 91, 161904 (2007).

${ }^{13}$ G. Koblmüller, F. Wu, T. Mates, J. S. Speck, S. Fernández-Garrido, and E. Calleja, Appl. Phys. Lett. 91, 221905 (2007).

${ }^{14}$ A. Pisch and R. Schmid-Fetzter, J. Cryst. Growth 187, 329 (1998).

${ }^{15}$ D. D. Koleske, A. E. Wickenden, R. L. Henry, M. E. Twigg, J. C. Culbertson, and R. J. Gorman, Appl. Phys. Lett. 73, 2018 (1998).

${ }^{16}$ T. Zywietz, J. Neugebauer, and M. Scheffler, Appl. Phys. Lett. 73, 487 (1998).

${ }^{17}$ J. Neugebauer, T. Zywietz, M. Scheffler, J. E. Northrup, H. Chen, and R. M. Feenstra, Phys. Rev. Lett. 90, 056101 (2003).

${ }^{18}$ G. Koblmüller, J. Brown, R. Averbeck, H. Riechert, P. Pongratz, and J. S. Speck, Jpn. J. Appl. Phys., Part 2 44, L906 (2005). 